\title{
The Relationship between Random Gap Detection and Hearing in Noise Test Performances
}

DOI: $10.3766 /$ jaaa. 18005

\author{
Paige Heeke* \\ Andrew J. Vermiglio* \\ Emery Bulla* \\ Keerthana Velappan* \\ Xiangming Fang $\dagger$
}

\begin{abstract}
Background: Temporal acoustic cues are particularly important for speech understanding, and past research has inferred a relationship between temporal resolution and speech recognition in noise ability. A temporal resolution disorder is thought to affect speech understanding abilities because persons would not be able to accurately encode these frequency transitions, creating speech discrimination errors even in the presence of normal pure-tone hearing.

Purpose: The primary purpose was to investigate the relationship between temporal resolution as measured by the Random Gap Detection Test (RGDT) and speech recognition in noise performance as measured by the Hearing in Noise Test (HINT) in adults with normal audiometric thresholds. The second purpose was to examine the relationship between temporal resolution and spatial release from masking.

Research Design: The HINT and RGDT protocols were administered under headphones according to the guidelines specified by the developers. The HINT uses an adaptive protocol to determine the signalto-noise ratio where the participant recognizes $50 \%$ of the sentences. For HINT conditions, the target sentences were presented at $0^{\circ}$ and the steady-state speech-shaped noise and a four-talker babble (4TB) was presented at $0^{\circ},+90^{\circ}$, or $-90^{\circ}$ for noise front, noise right, and noise left conditions, respectively. The RGDT is used to evaluate temporal resolution by determining the smallest time interval between two matching stimuli that can be detected by the participant. The RGDT threshold is the shortest time interval where the participant detects a gap. Tonal $(0.5,1,2$, and $4 \mathrm{kHz})$ and click stimuli random gap subtests were presented at $60 \mathrm{~dB}$ HL. Tonal subtests were presented in a random order to minimize presentation order effects.
\end{abstract}

Study Sample: Twenty-one young, native English-speaking participants with normal pure-tone thresholds ( $\leq 25 \mathrm{~dB}$ HL for $500-4000 \mathrm{~Hz}$ ) participated in this study. The average age of the participants was 20.2 years $(S D=0.66)$.

Data Collection and Analysis: Spearman rho correlation coefficients were conducted using SPSS 22 (IBM Corp., Armonk, NY) to determine the relationships between HINT and RGDT thresholds and derived measures (spatial advantage and composite scores). Nonparametric testing was used because of the ordinal nature of RGDT data.

Results: Moderate negative correlations $(p<0.05)$ were found between eight RGDT and HINT threshold measures and a moderate positive correlation $(p<0.05)$ was found between RGDT click thresholds and HINT 4TB spatial advantage. This suggests that as temporal resolution abilities worsened, speech recognition in noise performance improved. These correlations were not statistically significant after the $p$ value reflected the Bonferroni correction for multiple comparisons.

Conclusion: The results of the present study imply that the RGDT and HINT use different temporal processes. Performance on the RGDT cannot be predicted from HINT thresholds or vice versa.

*Department of Communication Sciences and Disorders, East Carolina University, Greenville, NC; †Department of Biostatistics, East Carolina University, Greenville, NC

Corresponding author: Paige Heeke, Department of Communication Sciences and Disorders, East Carolina University, Greenville, NC 27834; Email: heekep15@students.ecu.edu

Presented as a poster presentation at the American Academy of Audiology Conference in Indianapolis, IN, April 7, 2017. 
Key Words: speech recognition in noise, temporal resolution

\begin{abstract}
Abbreviations: GDT = gap detection threshold; GIN = Gaps-in-Noise; HINT = Hearing in Noise test; ILDs = interaural level differences; ITDs = interaural time differences; RGDT = Random Gap Detection Test; SD = standard deviation; SNR = signal-to-noise ratio; SSN = steady-state speech-shaped noise; $4 \mathrm{~TB}=$ four-talker babble
\end{abstract}

\section{INTRODUCTION}

$\mathrm{T}$ he purpose of the present study was twofold. The first objective was to investigate the relationship between temporal resolution as measured by the Random Gap Detection Test (RGDT; Keith, 2000) and speech recognition in noise performance as measured by the Hearing in Noise Test (HINT; Nilsson et al, 1994; Vermiglio, 2008) in adults with normal audiometric thresholds. The second objective was to examine the relationship between temporal resolution and spatial release from masking. The HINT was conducted in both steady-state speech-shaped noise (SSN) and a fourtalker babble (4TB).

\section{RGDT as a Measure of Temporal Resolution}

The RGDT was developed to identify and quantify timing disorders in the auditory system in adults and children (Keith, 2000). This test evaluates temporal resolution by determining the shortest time interval detectable between two stimuli separated by varying time intervals. In speech, transitions of formant frequencies occur in the 0-20 msec range. Abnormally long gap detection scores $(>20 \mathrm{msec})$ are, therefore, assumed to represent poorer than normal performance. A temporal resolution disorder in turn is thought to affect speech understanding abilities because persons would not be able to accurately encode these frequency transitions, creating speech discrimination errors even in the presence of normal pure-tone thresholds (Keith, 2000).

The RGDT is a measure of temporal integrity at the level of the cortex, with a low linguistic and cognitive load due to the simplicity of the task. It can be used for individuals with and without hearing loss and with any linguistic background. The RGDT has been used to evaluate temporal resolution in children with conductive hearing loss, auditory processing disorder, and auditory neuropathy. Children aged 7-10 years with conductive hearing loss and children with the diagnosis of a central auditory processing disorder showed RGDT thresholds significantly higher than normal children (Balen et al, 2009). Yalçınkaya et al (2009) investigated the performance of five children aged 7-13 years with auditory neuropathy and 10 normal hearing children aged 7-16 years who had not had an auditory processing problem as the control group. These children were unable to perform the RGDT and were subsequently tested with the expanded RGDT, which contains gaps between 50 and 300 msec. The auditory neuropathy group performed significantly poorer than the control group on the RGDT $(p<0.05)$. All children in the control group had gap detection thresholds (GDTs) within normal limits and all children with auditory neuropathy had GDTs that were considered abnormal. The abnormal temporal resolution in children with auditory neuropathy was most likely due to the poor neural synchrony of the auditory nerve.

\section{Speech Perception in Quiet and Temporal Resolution}

The auditory system encodes both temporal and spectral cues; however, temporal acoustic cues are particularly important for speech understanding (Phillips, 1999). Speech recognition performance was maintained when spectral information of speech was greatly reduced, requiring the participants to heavily rely on temporal cues (Shannon et al, 1995). The temporal distortion of speech (e.g., reverberant speech, interrupted speech, and rapid speech) results in poor speech perception (Gordon-Salant and Fitzgibbons, 1993). For individuals using cochlear implants, temporal modulation detection thresholds partially account for individual differences in speech recognition performance when presented at a comfortable listening level (Fu, 2002).

\section{Speech Perception in Noise and Temporal Resolution}

The presence of normal pure-tone thresholds does not guarantee the absence of perceived hearing difficulties, especially in the presence of background noise. It has been estimated that up to $12 \%$ of individuals with normal pure-tone thresholds have a self-reported hearing difficulty (Tremblay et al, 2015). Stephens et al (2003) reported that the prevalence of patients $<50$ years of age with normal pure-tone thresholds and the selfreport of speech recognition in noise deficits were $37 \%$. Past research has inferred a relationship between temporal resolution and speech recognition in noise. For example, Middelweerd et al (1990) investigated the speech recognition ability in individuals with normal pure-tone thresholds who complained of difficulty in understanding speech in noise (disordered group) and those who did not (control group). Speech recognition performance was measured in steady-state and fluctuating background noise. Participants were expected to 
perform better in fluctuating noise by taking advantage of the temporal dips in the background noise to improve understanding of the target speaker. In both steadystate noise and fluctuating noise conditions, the disordered group had significantly higher speech recognition thresholds than the control group ( $p<0.05$ ). The fluctuating noise results demonstrated that the disordered group was not able to take advantage of the temporal dips as much as the control group. This was attributed to impaired temporal resolution for the disordered group.

Tyler et al (1982) assessed the relationship between temporal resolution, measured by gap detection between two noise bursts, and speech in noise performance, measured by the four-alternative feature test. Longer GDTs were significantly associated with decreased performance on speech understanding in noise $(p<0.01)$, even with the effects of audiometric thresholds accounted for. Feng et al (2010) investigated the effects of high-frequency hearing loss on low-frequency temporal resolution. They hypothesized that although pure-tone hearing thresholds in the low-frequency region were within the normal range, low-frequency temporal resolution may contribute to difficulties understanding speech in noise. Temporal resolution was measured by a threealternative choice gap detection task in low-passed (with high-frequency cutoff of $1,2,3$, or $4 \mathrm{kHz}$ ) noise bursts with gap sizes varying from 1 to $2 \mathrm{msec}$. The HINT was used to evaluate speech perception in noise. The results of this study found that GDTs in low-passed noise bursts were poorer (longer) in individuals with high-frequency hearing loss compared with normal hearing controls. A significant positive correlation was seen between HINT performance and noise low-pass filtered at $1 \mathrm{kHz}$ GDTs $(p<0.05)$, where better performance on the HINT was associated with better GDTs.

Not all previous research has supported a relationship between temporal resolution and speech understanding in noise. For example, Hoover et al (2015) examined the relationship using the Gaps-in-Noise (GIN) test and the QuickSIN. They found that the GIN test scores did not explain the variance in QuickSIN performance once hearing loss and age were accounted for. These results were attributed to the GIN test being a measure of temporal resolution and sustained attention or processing speed. This is because of the setup of the GIN test in which a participant listens to continuous noise and must quickly respond by clicking a button. This requires vigilant attention of the participant and audiologist who is monitoring for responses, and it is presumed to allow for high chances of false positives and negatives.

\section{The Role of Temporal Resolution in the Spatial Release from Masking}

Spatial release from masking refers to the improvement in speech recognition in noise ability when the target speech signal is spatially separated from the noise. This is accomplished through the utilization of interaural time differences (ITDs) and interaural level differences (ILDs). According to Dubno et al (2002), ITDs have their greatest contribution in the low frequencies, whereas ILDs have their greatest contribution in the high frequencies. The authors evaluated contributions from ITDs and ILDs to speech understanding in noise. This was carried out by measuring performances on the HINT with both low- and high-pass filtered speech and noise in younger and older adults with normal hearing and older adults with mild-to-moderate sensorineural hearing loss. Younger and older adults showed benefit from the spatial separation of speech and noise when then the stimuli were low-pass filtered, indicating the use of ITDs. Older listeners with hearing loss also showed some benefit from spatial separation with the speech and noise low-pass filtered, but showed little to no benefit from spatial separation with the speech and noise high-pass filtered.

\section{Purpose}

The purpose of the present study was to investigate the relationship of temporal resolution and speech recognition in noise performance. Temporal resolution ability was determined with the RGDT and speech recognition in noise ability was determined by the HINT. This study appears to be the first to examine the relationship between the RGDT and HINT using steadystate noise and 4TB. Specifically, it was hypothesized that

- participant outcomes on the HINT and RGDT will be positively correlated, where better RGDT thresholds (smaller gaps) would be associated with better HINT thresholds (more negative signal-to-noise ratio [SNR]) and

- a negative relationship will be found between the RGDT and spatial advantage measures because of the temporal component involved in the spatial release from masking.

\section{METHODS}

$\mathrm{P}$ ermission to conduct this research study was obtained from the East Carolina University Institutional Review Board. Twenty-one young adults (20 females and one male) participated in this study. The average age was 20.2 years (standard deviation $[\mathrm{SD}]=0.62$ ) and the age range was from 19 to 22 years. Otoscopic visualization revealed clear ear canals for all participants. All participants were native English speakers with normal pure-tone thresholds $(\leq 25 \mathrm{~dB}$ HL for $500-8000 \mathrm{~Hz}$ ), with the exception of one who 
Table 1. Descriptive Statistics for HINT Quiet Thresholds (dBA) and All HINT Noise Thresholds (dB SNR)

\begin{tabular}{|c|c|c|c|c|c|c|c|c|c|}
\hline & $\begin{array}{l}\text { HINT } \\
\text { Quiet }\end{array}$ & $\begin{array}{l}\text { HINT SSN } \\
\text { Front }\end{array}$ & $\begin{array}{l}\text { HINT 4TB } \\
\text { Front }\end{array}$ & $\begin{array}{l}\text { HINT SSN } \\
\text { Left }\end{array}$ & $\begin{array}{l}\text { HINT 4TB } \\
\text { Left }\end{array}$ & $\begin{array}{c}\text { HINT SSN } \\
\text { Right }\end{array}$ & $\begin{array}{c}\text { HINT 4TB } \\
\text { Right }\end{array}$ & $\begin{array}{l}\text { HINT SSN } \\
\text { Composite }\end{array}$ & $\begin{array}{l}\text { HINT 4TB } \\
\text { Composite }\end{array}$ \\
\hline Mean & 27.33 & -1.86 & -1.22 & -8.55 & -9.97 & -8.8 & -10.53 & -5.27 & -5.74 \\
\hline SD & 4.80 & 1.04 & 1.21 & 1.38 & 1.69 & 1.23 & 1.59 & 0.92 & 1.03 \\
\hline Median & 26.9 & -2.0 & -1.6 & -9.0 & -10.3 & -8.70 & -10.3 & -5.33 & -5.83 \\
\hline$N$ & 21 & 21 & 21 & 21 & 21 & 21 & 21 & 21 & 21 \\
\hline Maximum & 37.2 & -0.1 & 1.6 & -5.7 & -6.5 & -6.1 & -8.4 & -3.33 & -3.93 \\
\hline Minimum & 16.7 & -3.5 & -2.6 & -10.6 & -12.6 & -11.2 & -13.1 & -6.83 & -7.55 \\
\hline Range & 20.5 & 3.4 & 4.2 & 4.9 & 6.1 & 5.1 & 4.7 & 3.50 & 3.63 \\
\hline
\end{tabular}

had thresholds of $30 \mathrm{~dB}$ HL for $6000 \mathrm{~Hz}$ in the left ear and $8000 \mathrm{~Hz}$ in both ears. This convenience sample was made up of undergraduate students in the Department of Communication Sciences and Disorders at East Carolina University.

The HINT was used to determine speech recognition in noise ability. The HINT sentences were also presented in quiet for comparison. The HINT was administered using custom software provided by the House Ear Institute in Los Angeles, CA. The speech and noise stimuli were presented in a simulated sound field under headphones using Knowles Electronics Mannequin for Auditory Research head-related transfer functions. The speaker locations in the simulated sound field environment were $1 \mathrm{~m}$ from the center of the head. Telephonics TDH$50 \mathrm{P}$ headphones (Huntington, NY) were used to deliver the stimuli. Short, simple American English sentences were presented in a masker presented at $65 \mathrm{dBA}$. Sentences were presented virtually at $0^{\circ}$, whereas the masker was presented from $0^{\circ}, 90^{\circ}$, and $270^{\circ}$ for noise front, noise right, and noise left conditions, respectively. The masking conditions included SSN and 4TB for all HINT conditions. The 4TB, obtained from Auditec, is the same source file for the masker used with the QuickSIN test (Auditec personal communication March 29, 2017; Killion et al, 2004). The participants were instructed to listen to and repeat each sentence heard in quiet or in noise. Using an adaptive protocol, the level of the sentences was varied based on the participant's response. A $4 \mathrm{~dB}$ step size was used for the first four sentences and a $2 \mathrm{~dB}$ step size was used for sentences five through 20. The HINT threshold is the SNR where a participant correctly recognizes 50\% of the sentences. The HINT composite score is the average of the thresholds for the noise front, noise right, and noise left conditions where the threshold for the noise front condition is weighted twice. The composite score equally weights the thresholds measured with and without spatial separation of the speech and noise stimuli. It is a single value used to represent the overall ability of the participants to recognize speech in noise. Spatial advantage is calculated by subtracting the noise side threshold from the noise front threshold.

The RGDT evaluates temporal resolution by determining the shortest detectible time interval between two matching stimuli ( 15 msec tone or $230 \mu$ sec click). This is the shortest stimulus interval found when the stimulus pairs are heard as two rather than as one. The stimuli were presented from a PC desktop computer through a Grason-Stadler GSI 61 audiometer (Eden Prairie, MN) to participants listening with Telephonics TDH-50P headphones at $60 \mathrm{~dB}$ HL (dial setting). Seven subtests were conducted in which gap intervals in the stimulus pairs varied between 0 and $40 \mathrm{msec}$ in either an ascending (practice/screening subtests only) or a random order. Tonal stimuli random gap subtests were presented in a random order to minimize presentation order effects.

Statistical analyses including descriptive and Spearman's rho statistics were conducted using SPSS 22. Nonparametric

Table 2. Descriptive Statistics for Mean Spatial Advantage (Noise Front Threshold Minus Noise Side Threshold) for HINT Noise and 4TB Conditions (dB)

\begin{tabular}{lcccccc}
\hline & $\begin{array}{l}\text { HINT SSN Spatial } \\
\text { Advantage NF-NL }\end{array}$ & $\begin{array}{c}\text { HINT SSN Spatial } \\
\text { Advantage NF-NR }\end{array}$ & $\begin{array}{c}\text { Avg. HINT SSN } \\
\text { Spatial Advantage }\end{array}$ & $\begin{array}{c}\text { HINT 4TB Spatial } \\
\text { Advantage NF-NL }\end{array}$ & $\begin{array}{c}\text { HINT 4TB Spatial } \\
\text { Advantage NF-NR }\end{array}$ & $\begin{array}{c}\text { Avg. HINT 4TB } \\
\text { Spatial Advantage }\end{array}$ \\
\hline Mean & 6.69 & 6.94 & 6.82 & 8.75 & 9.32 & 9.03 \\
SD & 1.46 & 1.32 & 1.28 & 1.31 & 2.12 & 1.47 \\
Median & 6.90 & 7.30 & 6.95 & 8.5 & 8.9 & 8.4 \\
N & 21 & 21 & 21 & 21 & 11.8 & 13.7 \\
Maximum & 9.4 & 9.8 & 9.5 & 6.4 & 6.6 & 7.1 \\
Minimum & 4.1 & 5.1 & 4.6 & 5.4 & 7.1 & 5.0 \\
Range & 5.7 & 4.7 & 4.9 & &
\end{tabular}

Note: $\mathrm{NF}=$ noise front threshold, $\mathrm{NL}=$ noise left threshold, $\mathrm{NR}=$ noise right threshold. 
Table 3. Descriptive Statistics for All RGDT Thresholds (ms)

\begin{tabular}{|c|c|c|c|c|c|c|c|c|}
\hline & $\begin{array}{c}\text { RGDT } \\
\text { Practice } \\
500 \mathrm{~Hz}\end{array}$ & $\begin{array}{c}\text { RGDT } \\
\text { Random } \\
500 \mathrm{~Hz}\end{array}$ & $\begin{array}{c}\text { RGDT } \\
\text { Random } \\
1000 \mathrm{~Hz}\end{array}$ & $\begin{array}{c}\text { RGDT } \\
\text { Random } \\
2000 \mathrm{~Hz}\end{array}$ & $\begin{array}{c}\text { RGDT } \\
\text { Random } \\
4000 \mathrm{~Hz}\end{array}$ & $\begin{array}{c}\text { RGDT } \\
\text { Random } \\
\text { Composite }\end{array}$ & $\begin{array}{c}\text { RGDT } \\
\text { Click } \\
\text { Practice }\end{array}$ & $\begin{array}{c}\text { RGDT } \\
\text { Click } \\
\text { Random }\end{array}$ \\
\hline$\overline{\text { Mean }}$ & 8.90 & 5.62 & 4.95 & 5.24 & 5.86 & 5.42 & 8.52 & 5.57 \\
\hline SD & 3.63 & 4.02 & 3.26 & 2.68 & 2.92 & 1.99 & 4.51 & 3.14 \\
\hline Median & 10 & 5 & 5 & 5 & 5 & 5.5 & 10 & 5 \\
\hline $\mathrm{N}$ & 21 & 21 & 21 & 21 & 21 & 21 & 21 & 21 \\
\hline Maximum & 15 & 15 & 15 & 10 & 10 & 10 & 20 & 8 \\
\hline Minimum & 2 & 2 & 2 & 2 & 2 & 2.75 & 2 & 2 \\
\hline Range & 13 & 13 & 13 & 8 & 8 & 7.25 & 18 & 10 \\
\hline
\end{tabular}

testing was used because of the ordinal nature of the RGDT data.

\section{RESULTS}

$\mathrm{T}$ able 1 shows the descriptive statistics for all HINT measures. The Quiet thresholds are expressed in $\mathrm{dBA}$ and the thresholds for the noise threshold measures are presented in $\mathrm{dB}$ SNR. The more negative the score, the better the performance. The average threshold for the SSN HINT noise front condition was $-1.86 \mathrm{~dB}$ SNR. This indicates that on average, the participants recognized half of the sentences when the speech was presented at $<1.86 \mathrm{~dB}$ level of the noise. The average SSN noise right and noise left thresholds were -8.55 and $-8.80 \mathrm{~dB}$ SNR, respectively. The average HINT SSN composite score was $-5.27 \mathrm{~dB}$ SNR. The average threshold for the HINT 4TB noise front condition was $-1.22 \mathrm{~dB}$ SNR. The average $4 \mathrm{~TB}$ noise right and noise left thresholds were -10.53 and $-9.97 \mathrm{~dB}$ SNR, respectively. The average HINT 4TB composite score was $-5.74 \mathrm{~dB}$ SNR. For HINT $4 \mathrm{~TB}$, the range of thresholds for the noise left condition was $6.1 \mathrm{~dB}$. Nilsson et al (1994) reported that every $1 \mathrm{~dB}$ change in HINT thresholds corresponds to a $10 \%$ change in intelligibility. Therefore, the $6.1 \mathrm{~dB}$ range corresponds to a predicted maximum change in intelligibility of $61 \%$ in this group of participants with normal pure-tone thresholds. There was an average $6.82 \mathrm{~dB}$ improvement in speech recognition in noise performance when the noise was spatially separated from the target speech (spatial advantage) in SSN and an average $9.03 \mathrm{~dB}$ improvement in 4TB. Table 2 shows descriptive statistics for the spatial advantage.

Table 3 shows the descriptive statistics for all RGDT measures. The RGDT threshold is the shortest time interval where the participant detects a gap and is expressed in ms. Median RGDT thresholds for 500, 1000,2000 , and $4000 \mathrm{~Hz}$ tone subtests were all $5 \mathrm{msec}$. The average RGDT composite tone threshold (average of thresholds for subsets $0.5,1,2$, and $4 \mathrm{kHz}$ ) was 5.50 msec and the median click threshold was 5 msec.

Tables 4 and 5 show the Spearman rho correlation coefficients and $p$ values for the RGDT versus HINT measures. Moderate negative correlations were found between RGDT $1000 \mathrm{~Hz}$ thresholds and HINT SSN noise front and HINT 4TB noise right. Moderate negative correlations were found between RGDT click thresholds and HINT SSN noise front, noise left, and composite score, and with HINT 4TB noise right, noise left, and composite score. A moderate positive correlation was found between RGDT click thresholds and HINT 4TB spatial advantage. Multiple comparisons were accounted for by using a Bonferroni corrected alpha value of 0.001 $(\alpha=0.05 / 66)$. No significant correlations were found between any HINT measure and RGDT measure after the $p$ value reflected the Bonferroni correction.

\section{DISCUSSION}

The goal of this study was to determine the rela1 tionship between performances on a measure of temporal resolution (RGDT) and a measure of speech recognition in noise (HINT). Based on previous studies,

Table 4. Spearman rho Correlation Coefficients between RGDT and HINT Quiet and SSN Conditions

\begin{tabular}{|c|c|c|c|c|c|c|}
\hline & HINT Quiet & HINT SSN Front & HINT SSN Right & HINT SSN Left & $\begin{array}{l}\text { HINT SSN } \\
\text { Composite }\end{array}$ & $\begin{array}{c}\text { Avg. HINT SSN } \\
\text { Spatial Advantage }\end{array}$ \\
\hline RGDT $500 \mathrm{~Hz}$ & $0.144(0.533)$ & $-0.021(0.928)$ & $0.415(0.062)$ & $0.406(0.068)$ & $0.281(0.217)$ & $-0.437(0.048)$ \\
\hline RGDT $1000 \mathrm{~Hz}$ & $-0.248(0.278)$ & $-0.507(0.019)$ & $-0.178(0.440)$ & $-0.180(0.435)$ & $-0.417(0.060)$ & $-0.230(0.316)$ \\
\hline RGDT $2000 \mathrm{~Hz}$ & $0.098(0.673)$ & $-0.047(0.840)$ & $0.403(0.070)$ & $0.085(0.716)$ & $0.142(0.540)$ & $-0.279(0.221)$ \\
\hline RGDT $4000 \mathrm{~Hz}$ & $0.143(0.536)$ & $-0.087(0.709)$ & $0.003(0.991)$ & $-0.017(0.943)$ & $-0.055(0.814)$ & $0.063(0.786)$ \\
\hline RGDT Composite & $0.056(0.808)$ & $-0.266(0.244)$ & $0.273(0.232)$ & $0.153(0.507)$ & $-0.001(0.996)$ & $-0.431(0.051)$ \\
\hline RGDT Click & $-0.353(0.117)$ & $-0.617(0.003)$ & $-0.365(0.104)$ & $-0.517(0.016)$ & $-0.669(0.001)$ & $-0.047(0.839)$ \\
\hline
\end{tabular}

Notes: The Spearman rho correlation coefficient $\left(r_{\mathrm{s}}\right) p$ values are shown. Statistically significant relationships appear in bold font. 
Table 5. Spearman rho Correlation Coefficients between RGDT and HINT 4TB Conditions

\begin{tabular}{lrrrrr}
\hline & HINT 4TB Front & HINT 4TB Right & HINT 4TB Left & HINT 4TB Composite & Avg. HINT 4TB Spatial Advantage \\
\hline RGDT $500 \mathrm{~Hz}$ & $0.265(0.246)$ & $0.027(0.584)$ & $0.162(0.484)$ & $0.271(0.235)$ & $0.056(0.808)$ \\
RGDT $1000 \mathrm{~Hz}$ & $-0.012(0.960)$ & $-\mathbf{0 . 4 3 7}(\mathbf{0 . 0 4 8})$ & $-0.273(0.230)$ & $-0.288(0.206)$ & $0.383(0.086)$ \\
RGDT 2000 Hz & $-0.270(0.236)$ & $0.094(0.686)$ & $-0.229(0.318)$ & $-0.216(0.346)$ & $-0.141(0.543)$ \\
RGDT 4000 Hz & $-0.067(0.471)$ & $0.329(0.145)$ & $0.072(0.757)$ & $0.059(0.80)$ & $-0.356(0.113)$ \\
RGDT Composite & $-0.023(0.921)$ & $0.037(0.873)$ & $-0.081(0.727)$ & $-0.032(0.889)$ & $0.008(0.974)$ \\
RGDT Click & $-0.127(0.582)$ & $-\mathbf{0 . 4 7 6}(\mathbf{0 . 0 2 9})$ & $-\mathbf{0 . 5 7 5}(\mathbf{0 . 0 0 6})$ & $-\mathbf{0 . 4 9 5}(\mathbf{0 . 0 2 3})$ & $\mathbf{0 . 4 8 2}(\mathbf{0 . 0 2 7})$ \\
\hline Notes: The Spearman rho correlation coefficient $\left(r_{\mathrm{s}}\right) p$ values are shown. Statistically significant relationships appear in bold font.
\end{tabular}

Notes: The Spearman rho correlation coefficient $\left(r_{\mathrm{s}}\right) p$ values are shown. Statistically significant relationships appear in bold font.

it was hypothesized that as HINT performance improves, temporal resolution measured by the RGDT improves. The results of this study do not support this hypothesis. Although the correlations were not statistically significant following Bonferroni correction for multiple comparisons, moderate correlations between some test results could not be ignored. Specifically, eight moderate negative correlations were found between RGDT measures and HINT threshold measures. This suggests that as temporal resolution abilities worsened, speech perception in noise performance improved. These results suggest that the temporal processing underlying the two tasks differ.

The RGDT was developed as a quick clinical measure for evaluating temporal resolution in which the participant would not be able to predict the next gap in the stimulus pairs. In each subtest, each silent gap interval is presented only once and, therefore, could be affected by any slight inattention by the participant. Hoover et al (2015) compared speech recognition in noise performance with the GIN test (Musiek et al, 2005) and a psychoacoustic GDT test with an adaptive tracking method. Both GIN scores and GDT scores were found to have a significant moderate positive correlation $(p<0.01)$ with QuickSIN scores. Although the GDT explained a small portion $(6 \%)$ of variability in speech in noise performance beyond that related to age and amount of hearing loss (63\%), the GIN did not. Therefore, more simplified clinical measures of temporal resolution may not be sensitive to temporal processing underlying speech in noise performance.

Phillips (1999) described the difference between "within-channel" gap detection and "between-channel" gap detection where acoustic features on either side of the gap affect whether the response reflects discontinuity detection in one group of neurons or relative timing of activity across different groups of neurons. In this case, the RGDT is representative of a "within-channel" gap detection test, whereas the HINT is representative of a "between-channel" gap detection task. The results of the present study imply that the RGDT and HINT use different temporal processes.

\section{Clinical Implications}

According to the results from young adults with normal pure-tone thresholds, performance on the RGDT do not have predictive value of performance on speech perception in noise performances using the HINT, or vice versa. In the present study, a number of correlations between HINT and RGDT performance suggest that better speech understanding in noise is associated with worse GDTs, which is puzzling in context of research that emphasizes the role temporal acoustic cues in speech understanding (Shannon et al, 1995; Phillips, 1999). Traditional psychoacoustic GDT tasks are better predictors of speech perception in noise performance, but they are time consuming, require psychoacoustic instrumentation and extensive training of the participant (Phillips, 1999). It may be appropriate to evaluate both speech recognition performance in noise and a clinically used measure of temporal resolution, such as the RGDT, to evaluate temporal processing in young adult participants with normal pure-tone thresholds.

\section{CONCLUSIONS}

D ast research suggests an association between temporal resolution performance and speech perception in noise performance (Tyler et al, 1982; Middelweerd et al, 1990) because of the role of temporal acoustic cues for speech understanding (Phillips, 1999). However, according to the study results for young adult participants, HINT performance cannot be predicted from RGDT performance. The lack of relationships between the two tests suggests that the temporal resolution abilities used by each task differ.

\section{REFERENCES}

Balen SA, Bretzke L, Mottecy CM, Liebel G, Boeno MRM, Gondim LMA. (2009) Temporal resolution in children: comparing normal hearing, conductive hearing loss and auditory processing disorder. Braz J Otorhinolaryngol 75(1):123-129.

Dubno JR, Ahlstrom JB, Horwitz AR. (2002) Spectral contributions to the benefit from spatial separation of speech and noise. J Speech Lang Hear Res 45(6):1297-1310.

Feng Y, Yin S, Kiefte M, Wang J. (2010) Temporal resolution in regions of normal hearing and speech perception in noise for adults with sloping high-frequency hearing loss. Ear Hear 31(1): 115-125.

Fu QJ. (2002) Temporal processing and speech recognition in cochlear implant users. Neuroreport 13(13):1635-1639. 
Gordon-Salant S, Fitzgibbons PJ. (1993) Temporal factors and speech recognition performance in young and elderly listeners. $J$ Speech Lang Hear Res 36(6):1276-1285.

Hoover E, Pasquesi L, Souza P. (2015) Comparison of clinical and traditional gap detection tests. J Am Acad Audiol 26(6):540-546.

Keith RW. (2000) Random Gap Detection Test. St. Louis, MO: Auditec of St Louis Ltd www.auditec.com.

Killion MC, Niquette PA, Gudmundsen GI, Revit LJ, Banerjee S. (2004) Development of a quick speech-in-noise test for measuring signal-to-noise ratio loss in normal-hearing and hearing-impaired listeners. J Acoust Soc Am 116(4):2395-2405.

Middelweerd MJ, Festen JM, Plomp R. (1990) Difficulties with speech intelligibility in noise in spite of a normal pure-tone audiogram: original papers. Audiology 29(1):1-7.

Musiek FE, Shinn JB, Jirsa R, Bamiou D-E, Baran JA, Zaidan E. (2005) GIN (gaps-in-noise) test performance in subjects with confirmed central auditory nervous system involvement. Ear Hear 26(6):608-618.

Nilsson M, Soli SD, Sullivan JA. (1994) Development of the hearing in noise test for the measurement of speech reception thresholds in quiet and in noise. J Acoust Soc Am 95(2): 1085-1099.
Phillips DP. (1999) Auditory gap detection, perceptual channels, and temporal resolution in speech perception. J Am Acad Audiol $10: 343-354$.

Shannon RV, Zeng FG, Kamath V, Wygonski J, Ekelid M. (1995) Speech recognition with primarily temporal cues. Science 270(5234): 303-304.

Stephens D, Zhao F, Kennedy V. (2003) Is there an association between noise exposure and King Kopetzky syndrome? Noise Health $5(20): 55-62$

Tremblay KL, Pinto A, Fischer ME, Klein BE, Klein R, Levy S, Tweed TS, Cruickshanks KJ. (2015) Self-reported hearing difficulties among adults with normal audiograms: the beaver Dam offspring study. Ear Hear 36(6):e290.

Tyler RS, Summerfield Q, Wood EJ, Fernandes MA. (1982) Psychoacoustic and phonetic temporal processing in normal and hearing-impaired listeners. J Acoust Soc Am 72(3):740-752.

Vermiglio AJ. (2008) The American English hearing in noise test. Int $J$ Audiol 47(6):386-387.

Yalçınkaya F, Muluk NB, Ataş A, Keith RW. (2009) Random gap detection test and random gap detection test-expanded results in children with auditory neuropathy. Int $J$ Pediatr Otorhinolaryngol 73(11):1558-1563. 